\title{
La complessità e la medicina di laboratorio
}

\section{Complexity and laboratory medicine}

\author{
P. Cappelletti
}

Ricevuto: 29 novembre 2014 / Accettato: 10 dicembre 2014

(C) Springer-Verlag Italia 2014

Riassunto La "complessità" può essere definita come un quadro interpretativo per la modellizzazione delle scienze della natura e della società. La "biologia dei sistemi" può essere definita come il tentativo di integrare l'insieme dei dati biologici individuali nel contesto di un sistema, per comprendere come i singoli componenti interagiscono nel tempo e nello spazio per determinarne il funzionamento, studiandoli a livelli (scale) multipli spaziali e temporali. La "medicina di sistema" si può definire come l'applicazione alla medicina clinica della System Biology.

La System Medicine considera l'individuo, sia esso sano sia malato, come un sistema complesso, strutturato in sistemi, organi, tessuti e cellule, in cui le singole parti che lo compongono interagiscono tra loro (fisiologicamente e fisiopatologicamente) in maniera dinamica con i determinanti non biologici e che può essere descritto attraverso un diagramma di contingenza in cui la valutazione e l'integrazione clinica omnicomprensiva vengono realizzate attraverso la valutazione dell'intero genoma, ivi inclusa la "gene-enviromnent interaction", per evidenziare i cluster significativi ai fini dell'individuazione degli aspetti prevalenti in un sistema complesso che mostra proprietà e regolarità "emergenti". La $S y$ stem Medicine ha stretti legami con la Medicina Personalizzata, anche se ne amplifica i mandati, con la 4Ps Medicine e con la Medicina di Precisione per indirizzare una nuova visione dell' organizzazione sanitaria e della salute pubblica. La Medicina di Laboratorio non può non affrontare il tema della complessità per almeno tre motivi: nel paziente complesso deve tenere conto delle interazioni derivanti dalla polipatologia e dalla polifarmacologia, l'emergere di nuove

P. Cappelletti $(\varangle)$

IRCCS CRO Aviano, Via Franco Gallini 2, 33081 Aviano, PN, Italia

e-mail: piero.cappelletti@cro.it sindromi "complesse" come la sindrome metabolica e il suo ruolo nella Medicina Traslazionale.

Parole chiave Complessità · Biologia dei sistemi . Medicina dei sistemi - 4Ps Medicine

Summary "Complexity" can be defined as a conceptual framework for the modeling in nature and society sciences. Systems biology is the computational and mathematical modeling of complex biological systems. System medicine is the development of computational models that describe disease progression and the effect of therapeutic intervention. While system biology is aimed at a fundamental understanding of biological processes and ultimately at an exhaustive modeling of biological network, system medicine emphasizes that the essential purpose and relevance of models is translational, aimed at diagnostic, predictive, and therapeutic applications. System Medicine is strictly related to Personalized Medicine, 4Ps Medicine, and Precision Medicine. The challenges of the complexity paradigm to Laboratory Medicine are in the fields of diagnostic interpretation of complex patients, emergent complex diseases as Metabolic Syndrome, and Translational Medicine.

Keywords Complexity - System biology - System medicine . 4Ps Medicine

\section{La complessità}

All' inizio del millennio Mark C. Taylor [1], filosofo e saggista americano, scrive nel suo fondamentale "The moment of Complexity": "Stiamo vivendo in un momento di complessità senza precedenti, dove tutto si trasforma più rapidamente 
di quanto riusciamo a comprendere. È una fase di transizione da un'epoca che appariva stabile a sicura a un' altra in cui, come tanti sperano, tornerà l'equilibrio... A caratterizzare il momento della complessità non è il cambiamento in sé, quanto il ritmo del cambiamento. Ogni cosa si muove sempre più rapidamente, fino a rendere la velocità un obiettivo in sé". In queste parole si ritrovano le riflessioni sulla caratteristica dei tempi attuali e della determinante tecnologica: l'accelerazione. Nel mondo che sta emergendo, la complessità è una condizione irriducibile quanto ineluttabile, che, se da una parte genera confusione e incertezza, dall'altra ci offre, nelle attuali trasformazioni sociali, economiche, politiche e culturali, la possibilità di ripensare noi stessi in modo nuovo, riflettendo sulla visione postmoderna del mondo [2].

Mentre "semplice" e "complicato" derivano dalla stessa radice indoeuropea "plek" (con pieghe e senza pieghe), "complesso" deriva, secondo Edgard Morin, da "complexus" cioè "tessuto insieme".

Oggi la "complessità" può essere definita [3] come un quadro interpretativo per la modellizzazione delle scienze della natura e della società, un approccio, un processo più che un teorema o una teoria generale - paragonabile alla traiettoria in meccanica, al livello atomico in fisica o al codice genetico in biologia — che prende atto dell'insufficienza del "riduzionismo", nato con Occam, nello spiegare fenomeni che appaiono come "sistemi complessi".

Le teorie e i modelli della complessità sono figli di studi interdisciplinari e le attuali modellizzazioni sono interdisciplinari per definizione: esse rappresentano il superamento del frazionamento e dello specialismo e l'apertura a una comprensione olistica dei fenomeni. La complessità può rappresentare un nuovo paradigma, caratterizzato da concetti quali feedback dinamico, non linearità, frattali, caos deterministico, autorganizzazione, fenomeni emergenti, tuttavia non kunhiano, perché è un nuovo pensiero dominante che si è affermato evolutivamente e carsicamente e non come rivoluzione sostitutiva delle teorie accompagnata da iperattività scientifica [3].

I sistemi complessi sono caratterizzati da relazioni diffuse tra le parti eterogenee che agiscono localmente le une sulle altre in uno spazio definito; sono privi di un controllore generale che governi o indirizzi l'evoluzione verso obiettivi predefiniti; possiedono un' organizzazione orizzontale più che gerarchica, con molti tipi di interazioni intersecanti; sono caratterizzati da un adattamento continuo per processi di evoluzione di singole parti (CAS). Inoltre, sono soggetti a instabilità di moto e fenomeni di biforcazione; la medesima legge deterministica può dare origine a grande varietà di possibili dinamiche, compreso il caos; le strutture frattali trovano applicazione nella descrizione delle dinamiche; le loro dinamiche sono in stati lontani dall'equilibrio (disequilibrio), ma possono comprendere molti stati di equilibrio o nessun equilibrio. Sottoposti a perturbazioni esterne reagiscono creando dinamiche nuove endogene non lineari e a priori del tutto imprevedibili e ingovernabili; producono forme di autorganizzazione e fenomeni emergenti (non spiegabili dalle parti), comprensibili cioè solo in un quadro olistico, non riduzionistico.

\section{La nascita della complessità}

La data di nascita della complessità è, di solito, posta nel 1948, quando Warren Weaver, uno dei padri della cibernetica, scrive l'articolo "Science and Complexity" sulla rivista di divulgazione scientifica American Scientist. Il riconoscimento accademico segue l'esplosione degli anni Settanta: i primi istituti dedicati sono il Santa Fe Institute dell'Università del New Mexico nel 1984 e il Center for Complex System Research dell'Università dell'Illinois nel 1986. Tuttavia, nella concettualizzazione attuale della complessità si raccolgono, in effetti, riflessioni e teorie elaborate a partire dal tardo Ottocento.

Secondo Bertuglia e Vaio [3], le teorie prodromiche sono rinvenibili, da un lato, nell'evoluzione del concetto di caos in fisica e in matematica, che fa la prima comparsa negli ultimi decenni del XIX secolo con Hadarmad, Poincarè e Birkhof, da cui derivano le ricerche di Lorenz (1963) sull'attrattore come spazio che intrappola le traiettorie (di lui è noto il cosiddetto "effetto farfalla"), quelle di Mandelbrot (1975) sulla geometria frattale per spiegare l'autosomiglianza (somiglianza a differenti scale) e quelle di Thom (1967) sulla teoria delle catastrofi (biforcazioni) per spiegare la morfogenesi. Dall'altro lato è fondamentale l'evoluzione del concetto di autorganizzazione, che emerge in biologia nella teoria dell'adattamento di Lamark e nei lavori di Thompson che nel 1917 scrive "On Growth and Form" (dove nasce il moderno concetto della relazione forma-funzione) e nell'economia nei lavori di von Mises e von Hayek (1933). In chimica l'autorganizzazione è una caratteristica risposta nei sistemi dissipativi, lontani dall'equilibrio "on edge of chaos", secondo Prigogine, premio Nobel per la chimica nel 1977. È riferendosi a queste teorie che Haken scopre il modello di laser non lineare, fenomeno auto-organizzativo di treni d'onda cooperativi in situazioni lontane dall'equilibrio, e dà origine alla riflessione teorica della "sinergetica" come euristica per modelli complessi basata sui principi dell'asservimento e dei parametri d'ordine (in ciò collegandosi alla cibernetica). Negli anni Ottanta il concetto viene riproposto nei termini di "autopoiesi" (sintesi di autorganizzazione ed evoluzione) in biologia (la cellula) da Maturano e Varela e negli studi sociali da Luhmann (1984).

Infine, un terzo gruppo di teorie, imparentate con il concetto di autorganizzazione ma che fanno riferimento ai problemi della comunicazione, si sviluppano dal dopoguerra in poi: la cibernetica (scienza del controllo e della comunicazione) con gli studi sulla retroazione (feedback) che con von 
Neuman prende la strada del "problem solving" e delle macchine costruite allo scopo e con Asley quella dello studio dell' "omeostasi" a sostenere l'esplosione dell'intelligenza artificiale, della robotica, dell'automatica, della bionica; la scienza dell'informazione con Wiener, altro padre della cibernetica, e con Shannon, creatore della teoria dell'informazione (con i concetti di rumore e ridondanza) e inventore del bit; l'epistemologia cibernetica come meta-scienza di Bateson (1972), che parla di mente e corpo come software e hardware, del "sistema mente" come un tutto e della profonda connessione mente-natura in un unico gigantesco sistema: "la creatura".

I tentativi di teorie generali si sono succeduti con la Teoria Generale del Sistema (1968) di von Bertalanffy, biologo che vi giunge attraverso una teoria dei sistemi organici (in disequilibrio e organizzati, omeostatici) e una teoria dei sistemi aperti (negentropici) e approda a una teoria unificante che vede i sistemi come qualcosa più delle loro parti e in grado di autoripararsi, sistemi che appartengono tanto agli organismi viventi, quanto ai sistemi sociali e alle macchine cibernetiche.

Dopo la nascita e l'affermazione della cibernetica, è Le Moigne (1977) che tenta di definire una teoria unificante, piuttosto come una Teoria del Sistema Generale, dove i sistemi sono meta-modelli, cioè strumenti di modellizzazione più che oggetti di modellizzazione.

Infine Morin, nel suo "La methode" (1977-2004) fonda il pensiero complesso, che passa per più piani: il primo costituito dalla teoria dell'informazione (rumore/ridondanza), dalla cibernetica (teoria delle macchine) e dalla teoria dei sistemi (organizzazione: il tutto è più delle parti); il secondo dalle idee sull' "emergenza" dei fenomeni nei sistemi; infine un terzo piano definito dialogico (contiene i contrari), ricorsivo (processi contemporaneamente cause ed effetto) e ologrammatico (la parte è nel tutto, ma anche il tutto è nella parte, come in un ologramma).

Nel percorso teorico della complessità, la modellizzazione (modeling) ha percorso sostanzialmente tre traiettorie: le reti neurali e simulazione dei processi mentali (intelligenza artificiale; linguistica; teorie economiche); gli automi cellulari per simulazioni di sistemi spaziali (scienze urbane e regionali); i sistemi multiagente come paradigma computazionale per progettare software (swarm intelligence; sistemi di persone). La misura della complessità, infine, è costitutivamente difficile perché la complessità è "percepita", è percepita selettivamente e può essere solo messa in relazione con la quantità minima di informazione capace di descrivere il sistema. I metodi proposti sono fondamentalmente la misura dell'informazione di Shannon, la complessità di Kolmogorov e la complessità computazionale.

\section{System biology}

Wikipedia [4] definisce la biologia dei sistemi-uno dei più caldi ed elusivi campi della biologia [5]_-"Systems biology (also known as Systeomics) is the computational and mathematical modeling of complex biological systems", in ciò sottolineando l'obiettivo della comprensione della complessità del vivente, la costitutiva interdisciplinarietà della nuova disciplina [6] e i metodi utilizzati quali la modellizzazione computazionale, riprendendo la definizione di uno dei padri della "computational system biology" Hiroshi Kitano [7]. La "biologia dei sistemi" può essere definita, inoltre, come il tentativo di integrare l'insieme dei dati biologici individuali nel contesto di un sistema, per comprendere come i singoli componenti interagiscano nel tempo e nello spazio per determinarne il funzionamento, studiandoli a livelli (scale) multipli spaziali e temporali [8]. Gli approcci sono molteplici e possono sostanzialmente essere distinti in: "hypothesis-driven" più tradizionale, per rivelare comunque proprietà emergenti, che richiede dati quantitativi multi-scala spaziali e temporali, la costruzione di modelli di conoscenza e modelli computazionali e che può essere applicata a sistemi viventi per scoprire proprietà quali network di geni, "signaling cascade", network metabolici, popolazioni o ecosistemi; "hypothesis-free" (non-biased) associato con il più recente sviluppo dei metodi ad alta produttività ("omics") che utilizza i "data set" completi degli elementi cellulari in determinate condizioni, potenzialmente rivela nuovi fenomeni e può indurre nuove ipotesi e per corrette interpretazioni richiede un "high-throughput data processing", l'integrazione con una "comprensione" biologica e la modellizzazione. Essi sono riferiti anche come "bottom-up" e "top-down" [9].

Le ragioni della nascita della biologia dei sistemi sono comunemente riferite, da un lato, al successo delle bioscienze molecolari, spinto dalla bioinformatica, dalle "omics" e dagli strumenti "high-throughput", e alla conseguente necessità di comprendere la grande messe di dati a disposizione spostando il focus dalle molecole alle loro interazioni ("network") e, dall'altro, dall'insufficienza del riduzionismo per la comprensione della "nuova" realtà [10].

Per quanto riguarda quest'ultimo punto occorre sottolineare [11] come la fine del riduzionismo interteoretico affermatosi tra gli anni Trenta e Sessanta del secolo scorso non possa dare spazio a un olismo retorico e vagamente spiritualista come quello nato negli anni Venti. Piuttosto si va affermando, da un lato, una sorta di neo-riduzionismo che abbandona i miti novecenteschi in favore di una visione evoluzionaria e, dall'altro, una sorta di neo-olismo denominato "relational biology" privato del vitalismo e puntato ai network, in grado di costituire una teoria di riferimento per l'interpretazione della complessità del vivente. 
Tabella 1 Caratteristiche delle tre generazioni di medicina basata sulle omiche (modificata da [14])

\begin{tabular}{|c|c|c|c|c|c|}
\hline Generazione & Nome & Informazioni & Obiettivi & Informatica & Metodi \\
\hline $1^{\mathrm{a}}$ & Medicina genomica & $\begin{array}{l}\text { Mutazioni e } \\
\text { polimorfismi di } \\
\text { cellule germ-free }\end{array}$ & $\begin{array}{l}\text { Medicina } \\
\text { personalizzata }\end{array}$ & Approccio statistico & $\begin{array}{l}\text { Genetica statistica, } \\
\text { farmacogenetica, } \\
\text { farmacogenomica }\end{array}$ \\
\hline $2^{\mathrm{a}}$ & $\begin{array}{l}\text { Medicina } \\
\text { post-genomica }\end{array}$ & $\begin{array}{l}\text { Profili molecolari } \\
\text { globali di cellule } \\
\text { somatiche }\end{array}$ & $\begin{array}{l}\text { Medicina predittiva } \\
\text { e preventiva }\end{array}$ & $\begin{array}{l}\text { Approccio guidato } \\
\text { dai dati }\end{array}$ & $\begin{array}{l}\text { Data-mining, } \\
\text { machine-learning, } \\
\text { statistica esplorativa }\end{array}$ \\
\hline $3^{\mathrm{a}}$ & Medicina di sistema & $\begin{array}{l}\text { Percorsi e reti } \\
\text { molecolari e loro } \\
\text { alterazioni in } \\
\text { malattia }\end{array}$ & $\begin{array}{l}\text { Medicina } \\
\text { personalizzata, } \\
\text { predittiva, } \\
\text { preventiva e } \\
\text { partecipata } \\
\text { (4Ps Medicine) }\end{array}$ & $\begin{array}{l}\text { Approccio guidato } \\
\text { dai modelli }\end{array}$ & $\begin{array}{l}\text { Reverse } \\
\text { engineering, } \\
\text { simulazioni, design } \\
\text { di sistemi }\end{array}$ \\
\hline
\end{tabular}

Per il primo punto, una recente schematizzazione dei rapporti tra genomica funzionale (come "gene expression profiling", metabolomica e proteomica) e i diversi approcci di "systems-level analysis" da essa derivati come Gene Regulatory Network (GRN) inference, Pathway Analysis (PA) and Flux Balance Analysis (FBA), dei modelli di sistemi biologici così costruiti e interpretati ed espansi a un livello superiore dalla System Biology e i rapporti con la System Medicine, è stata recentemente prodotta da Conesa e Mortazavi [12].

Secondo Barabási et al [13] l'utilizzo della "System Biology" in medicina produce la "network medicine", dove il comportamento biologico non è il risultato del tradizionale lineare processo dei componenti strutturali e funzionali (identificati da genomica, proteomica e metabolomica), ma emerge dall'interazione di network biologici modulari scale-free composti dagli stessi componenti a molti diversi livelli: geni, transcritti, proteine, metaboliti, organelli, cellule, organi e organismi. E, secondo Tanaka [14], vi è un susseguirsi di generazioni dalla genomica alla "System Biology", che fanno riferimento a informazioni progressivamente più pregnanti (dai polimorfismi delle cellule germ-free ai profili molecolari delle cellule somatiche, ai percorsi e network), utilizzano sempre più raffinati approcci informatici (statistici classici, data-driven, model-driven) e metodi (genetica e genomica, data mining e learning machine, system simulation e design) e consentono obiettivi medici sempre più ambiziosi: medicina personalizzata, medicina predittiva, " $4 P s$ Medicine" (Tab. 1).

\section{System medicine}

Charles Auffray [15] può quindi definire la System Medicine come l'applicazione alla medicina clinica della System Biology. L'approccio "di sistema" alla biologia concettualizza l'ambito disciplinare della System Biology, che, applicata come strumento conoscitivo e integrativo, forte di strumenti computazionali avanzati e in stretta connessione con la computer science, ai domini fisiologici ed eziopatogenetici, definisce la System Medicine, in grado di comprendere il Fenotipo Complesso (o Fenoma). L'integrazione dei due momenti è tema di molte iniziative, anche italiane [16].

La Medicina clinica come la conosciamo da Ippocrate si fonda su due presupposti: la malattia, ontologia creata sulla base del consenso tra clinici, che guida l'azione del medico che la identifica-secondo il metodo clinico-e ne tenta la cura con i rimedi conosciuti e la diagnosi che è il riconoscimento di un pattern attraverso i punti di identità tra malattia-ontologia e quadro clinico, in un momento idiografico. Oggi, però, siamo di fronte a pazienti complessi con multimorbilità e/o comorbilità e polifarmacologia, sempre interconnesse con le variabili di acuzie e cronicità e con il livello di intensità di cura necessario. Ciò riguarda il 25\% della popolazione, prevalentemente gli anziani, ma la disabilità è presente in tutte le fasi della vita e si parla di paziente pediatrico complesso. Sono pazienti incomprensibili con il metodo clinico della parsimonia (individuazione di un costrutto e di una spiegazione monofattoriale) e con l'aiuto del solo livello di indagine individuale clinica (semeiotica). Il malato complesso viene assimilato, in maniera figurata, a una rete composta da nodi e fili di unione, in cui non tutti i nodi sono in connessione tra loro con la stessa quantità e qualità di interazioni [17].

È necessaria una "nuova" Medicina, che superi il riduzionismo clinico ed eziopatologico. L'approccio integrativo di modellizzazione della complessità clinica è sostenuto da un approccio di modellizzazione matematica e computazionale in biomedicina, mirante a comprendere globalmente il "sistema biologico", attraverso la definizione, più che delle singole parti componenti, delle loro integrazioni ed "emergenze", nei loro collegamenti dinamici e sinergici. I modelli sono open end, ovvero in continua definizione grazie all' acquisizione di progressive informazioni che attraverso appro- 
priati algoritmi prendono forma in cluster a significatività crescente [18].

La System Medicine, dunque, considera l'individuo, sia esso sano sia malato, come un sistema complesso, strutturato in sistemi, organi, tessuti e cellule, in cui le singole parti che lo compongono interagiscono fra loro (fisiologicamente e fisiopatologicamente) in maniera dinamica con $\mathrm{i}$ determinanti non biologici, che può essere descritto attraverso un diagramma di contingenza (contingency diagram) in cui la valutazione e l'integrazione clinica omnicomprensiva vengono realizzate attraverso la valutazione dell'intero genoma, ivi inclusa la "gene-enviromnent interaction" per evidenziare i cluster significativi ai fini dell'individuazione degli aspetti prevalenti in un sistema complesso che mostra proprietà e regolarità "emergenti" [17]. Per il riduzionismo il comportamento di un sistema biologico può essere spiegato dalle proprietà delle sue parti costituenti; le metafore utilizzate sono la macchina o la pallottola magica; si può identificare una singola causa efficiente dei fenomeni sperimentali; i fattori critici sono i predittori; i modelli sono lineari, prevedibili e spesso deterministici; la salute è normalità, riduzione del rischio e omeostasi. Per la biologia o medicina dei sistemi, il sistema biologico possiede proprietà emergenti che derivano dall'interrelazione e interazione delle parti e non appartengono a nessuna di esse singolarmente; la metafora è la rete (network); non esiste una causa unica dei fenomeni, ma molti fattori dinamici determinano il tutto; i fattori critici sono il contesto, tempo e spazio; i modelli sono non-lineari, sensibili alle condizioni iniziali, stocastici e caotici; la salute è robustezza, adattamento e plasticità, omeodinamica [19].

La System Medicine ha stretti legami con la Medicina Personalizzata, anche se ne amplifica i mandati [20, 21], con la "4Ps Medicine" e con la Medicina di Precisione per indirizzare una nuova visione dell'organizzazione sanitaria e della salute pubblica [22]. La 4Ps Medicine può essere definita come la medicina di sistema, cioè la personalizzazione dell'assistenza sanitaria in grado di conciliare le differenze individuali durante tutte le fasi del processo (prevenzione, diagnosi, trattamento, follow-up) [21]. La Medicina di Precisione è l'indagine delle caratteristiche patologiche individuali e istantanee, considerando i fattori genetici e biologici, epigenetici e ambientali, stili di vita e psicocognitivi (" $5 P S$ Medicine") [23].

\section{Complessità e medicina di laboratorio}

Il ruolo della Medicina di Laboratorio in questo quadro è già stato descritto [19, 21]. La Medicina di Laboratorio non può non affrontare il tema della complessità e nel concreto per almeno tre motivi: (a) nel paziente complesso la Medicina di Laboratorio deve tenere conto delle singole patologie, delle interazioni e degli effetti collaterali sulle diverse funzionalità d'organo che la polifarmacologia può determinare (esempi: valutazione della funzionalità renale (eGFR), del metabolismo del glucosio (glicemia, $\mathrm{HbA}_{1 \mathrm{c}}$ ), della funzione emopoietica (anemia), dell'assetto lipidico, della funzione cardiaca (PNC), della funzione epatica) [17]; (b) l'emergere di nuove sindromi "complesse" come per esempio la sindrome metabolica, in cui l'insulino-resistenza appare sempre meno come l'unica spiegazione e dove il rischio non è la semplice somma/moltiplicazione aritmetica dei rischi dei singoli fattori [21]; infine, il suo ruolo nella Medicina Traslazionale, in particolare per la diffusione delle "omics" e la validazione dei biomarcatori [24].

Conflitto di interesse Nessuno.

\section{Bibliografia}

1. Taylor CM (2001) The moment of complexity. Emerging network culture. The University of Chicago Press, Chicago

2. Cappelletti P (2012) Medicina di laboratorio e postmodernità. Riv Ital Med Lab 8:1-15

3. Bertuglia CS, Vaio F (2011) Complessità e modelli. Un nuovo quadro interpretativo per la modellizzazione nelle scienze della natura e della società. Bollati Boringhieri, Torino

4. http://en.wikipedia.org/wiki/System_biology (Accesso 27 ottobre 2014)

5. Pennisi E (2003) Tracing life's circuitry. Science 302:1646-1649

6. Friboulet A, Thomas D (2005) System biology-an interdisciplinary approach. Biosens Bioelectron 20:2404-2407

7. Kitano H (2001) Foundations of system biology. MIT Press, Cambridge

8. Coveney PV, Fowler PW (2005) Modelling biological complexity: a physical scientist's perspective. J R Soc Interface 2:267-280

9. Bruggeman FJ, Westerhoff HV (2007) The nature of system biology. Trends Microbiol 15:45-50

10. Strange K (2005) The end of "naïve reductionism": rise of systems biology or renaissance of physiology? Am J Physiol Cell Physiol 288:C968-C974

11. Gatherer D (2010) So what do we really mean when we say that system biology is holistic? BMC Syst Biol 4:22-34

12. Conesa A, Mortazavi A (2014) The common ground of genomics and system biology. BMC Syst Biol 8(Suppl 2):51-61

13. Barabási AL, Gulbahce N, Loscalzo J (2011) Network medicine: a network-based approach to human disease. Nat Rev Genet 12:5668

14. Tanaka H (2010) Omics-based medicine and system pathology. Methods Inf Med 2:173-185

15. Auffray C, Chen Z, Hood L (2009) System medicine: the future of medical genomics and healthcare. Genome Med 1:2

16. Clermont G, Auffray C, Moreau Y et al (2009) Bridging the gap between system biology and medicine. Genome Med 1:88

17. Ministero della Salute (2013) Quaderni del Ministero della Salute-Criteri di appropriatezza clinica, tecnologica e strutturale nell'assistenza del paziente complesso. http://www. quadernidellasalute.it/quaderni-flipbook/23-settembre-ottobre2013/index.php (Accesso 27 ottobre 2014)

18. Bousquet J, Anto JM, Sterk PJ et al (2011) Systems medicine and integrated care to combat chronic noncommunicable diseases. Genome Med 3:43

19. Cappelletti P (2010) La medicina di laboratorio predittiva, preventiva, personalizzata e partecipata. Il caso della sindrome metabolica. RIMeL/IJLaM 6(Suppl):6-10 
20. Offit K (2011) Personalized medicine: new genomics, old lessons. Hum Genet 130:3-14

21. Cappelletti P (2009) La medicina personalizzata tra ricerca e pratica clinica: il ruolo della medicina di laboratorio. RIMeL/IJLaM 5(Suppl):26-32

22. Burke W, Trinidad SB (2011) Systems medicine and the public's health. Genome Med 3:47
23. Gorini A, Pravettoni G (2011) P5 medicine: a plus for a personalized approach to oncology. Nat Rev Clin Oncol 8:444

24. Cappelletti P (2013) Innovazione ed evidenze in medicina di laboratorio. Riv Ital Med Lab 9:177-184 